\title{
The Association of Prescriber Awareness of Opioid Consumption Trends with Postoperative Opioid Prescription Volume in Hip Arthroscopy: Prescriber Awareness of Opioid Consumption
}

\author{
Daniel J. Cunningham, M.D., M.H.Sc., Richard C. Mather, M.D., M.B.A., \\ Steven A. Olson, M.D., and Brian D. Lewis, M.D.
}

\begin{abstract}
Purpose: To evaluate the impact of prescriber knowledge of 6-week postoperative opioid usage trends on postoperative opioid prescribing in hip arthroscopy for femoroacetabular impingement syndrome. Methods: Two groups of patients undergoing hip arthroscopy for femoroacetabular impingement syndrome with the same 2 surgeons were defined. One group preceded study design and implementation and 1 group was after study completion termed the preawareness group $(\mathrm{n}=129)$ and awareness group $(\mathrm{n}=130)$. Baseline clinical and operative characteristics and cumulative 6 -week postoperative opioid prescription amount in oral morphine equivalents (OMEs), initial discharge OMEs, and cumulative 6-week postoperative opioid refills were recorded. Multivariable models were constructed to evaluate the impact of provider awareness of opioid usage along with the other baseline characteristics previously mentioned on the outcomes of postoperative opioid prescribing. Results: Preawareness group (365.8 additional OMEs; $95 \%$ confidence interval [CI], 132.6-599; $P=.002$ ), preoperative opioid usage (506.2 additional OMEs; $95 \% \mathrm{CI}, 268.0-744.3 ; P<.001$ ), postoperative nonsteroidal anti-inflammatory drugs $(-664.6$ additional OMEs; -1002.6 to $-326.6 ; P<.001)$, and Caucasian race $(-597.5$ additional OMEs; $95 \%$ CI, -914.8 to $-280.2 ; P<.001)$ were significantly associated with 6 -week postoperative opioid prescribing. Caucasian race (odds ratio, $0.4 ; 95 \% \mathrm{CI}, 0.18-0.86 ; P=.02$ ) was associated with lower odds of additional postoperative opioid prescriptions whereas preoperative opioid usage (odds ratio, 2.47; 95\% CI, 1.4-4.36; $P=.002$ ) was associated with increased odds of additional postoperative opioid prescriptions. Conclusions: Patients in the awareness group received significantly lower opioid volume without an increase in overall prescription numbers. Level of Evidence: III, prognostic, retrospective comparative study.
\end{abstract}

S ymptomatic femoroacetabular impingement (FAI) syndrome is a source of pain in the hip that involves varying degrees of synovitis, labral damage, and bony impingement. ${ }^{1}$ Hip arthroscopy is an increasingly used modality ${ }^{2,3}$ that addresses this pathology providing short-term ${ }^{4}$ and sustained clinical benefit. ${ }^{5}$ Opioids may be part of a multimodal postoperative pain management strategy after hip arthroscopy. However, the United States is in the midst of an opioid use and abuse crisis. ${ }^{6-8}$ Although there are now several

From the Department of Orthopaedic Surgery, Duke University Medical Center, Durham, North Carolina, U.S.A.

The authors report the following potential conflicts of interest or sources of funding: R.M. is a paid consultant for KNG Health Consulting and Stryker, has received research support from Reflexion Health and Zimmer, and is a board or committee member of the Arthroscopy Association of North America and the North Carolina Orthopaedic Association. S.O. is a board or committee member of the Orthopaedic Trauma Association. Full ICMJE author disclosure forms are available for this article online, as supplementary material. studies in hand surgery and adult reconstruction suggesting appropriate opioid prescription targets, ${ }^{9-11}$ there is little information on appropriate opioid dosing after many other orthopaedic surgeries, including hip arthroscopy. Lack of knowledge may lead to overprescribing, which leads to an increased volume of unused opioid in the community that can contribute to opioid abuse. ${ }^{12-14}$ Further, the impact of knowledge of anticipated opioid consumption on prescriber practices is largely unknown.

Received December 8, 2019; accepted May 8, 2020.

Address correspondence to Daniel J. Cunningham, M.D., M.H.Sc., Box 3000,Durham, NC 27710.E-mail: daniel.cunningham@duke.edu

(C) 2020 THE AUTHORS. Published by Elsevier Inc. on behalf of the Arthroscopy Association of North America. This is an open access article under the CC BY-NC-ND license (http://creativecommons.org/licenses/by-nc-nd/4.0/).

2666-061X/191465

https://doi.org/10.1016/j.asmr.2020.05.004 
Formal education programs in hand surgery have been associated with significant reductions in postoperative opioid prescribing. ${ }^{15}$ A separate study found that patients undergoing upper extremity procedures used 5 to 15 oxycodone $5-\mathrm{mg}$ pills on average, and that patients had been overprescribed 3 times the amount of opioid that they actually used. ${ }^{9}$ Colleagues in general surgery have suggested procedure-specific opioid prescribing guidelines for common surgical procedures based on expert consensus in which the maximum amount recommended was 20 pills of oxycodone $5 \mathrm{mg} .{ }^{16}$

The authors of the current study previously reported results of a prospective, observational study of outpatient opioid pain medication usage in patients undergoing hip arthroscopy for FAI syndrome. ${ }^{17}$ This study demonstrated that $80 \%$ of patients without preoperative opioid usage consumed 30 oxycodone 5 -mg pills ( 225 oral morphine equivalents) or less in the 6-week postoperative period. The study also highlighted considerable overprescribing for most patients (approximately 50 unused oxycodone 5 -mg pills per patient). The greatest risk factor for increased postoperative usage was pre-operative usage, which increased postoperative usage 4-fold.

The purpose of this study is to evaluate the impact of prescriber knowledge of 6-week postoperative opioid usage trends on postoperative opioid prescribing in hip arthroscopy for FAI. The hypothesis of this study is that prescribers would reduce the volume of opioids prescribed at discharge and within 6 weeks postoperatively after obtaining knowledge regarding expected postoperative opioid usage. A secondary hypothesis is that the number of prescriptions would increase as prescribers reduced the volume of postoperative opioids prescribed.

\section{Methods}

\section{Study Design}

This is a single-center, retrospective, institutional review board-approved, comparative study of opioid prescribing patterns in patients undergoing hip arthroscopy (Current Procedural Terminology codes 29914 and/or 29916) for FAI syndrome before and after prescribers were aware of postoperative opioid usage trends. This study is designed and reported in accordance with the STROBE guidelines. ${ }^{18}$

\section{Variables and Data Sources}

Two groups of patients undergoing hip arthroscopy were identified according to whether or not their surgery was before or after the observational study of opioid usage in hip arthroscopy. The "preawareness" group was defined as patients undergoing surgery from December 2014 to May 2015. The "awareness" group was defined as patients undergoing surgery from October 2017 to March 2018. The primary study outcomes were cumulative oral morphine equivalents (OMEs) prescribed up to 6 weeks postoperatively, initial prescription size, and cumulative 6-week postoperative refills. Baseline and treatment characteristics included age, sex, race, current smoking, body mass index (BMI), American Society of Anesthesiologists score, active preoperative opioid usage as defined by active medication status at time of surgery, laterality, revision status, operative time, resident involvement in opioid prescribing at time of discharge, postoperative aspirin prescription, and postoperative nonsteroidal antiinflammatory drug (NSAID) prescription. Data were extracted through chart review.

\section{Intervention}

Two surgeons (S.O. and M.D.; R.M. and M.D.) and their advanced practice providers (J.B., nurse practitioner, and A.M., physician assistant) participated in the previously mentioned prospective observational study of opioid consumption in patients undergoing hip arthroscopy. Prescribing practices for patients undergoing surgery with these same 2 surgeons (S.O. and R.M.) were evaluated before study conception (preawareness group from December 2014 to May 2015) and after study submission for publication once results were well-known to the surgeons and their advanced practice providers (awareness group from October 2017 to March 2018).

\section{Missing Data}

All patients undergoing these procedures during this timeframe were included in the analysis. Three patients were missing data on BMI. Analyses were performed with and without inclusion of these patients, and there were no changes in study inference. Multivariable analyses excluding patients with missing data on BMI are presented.

\section{Statistical Analysis}

Sample size calculations were performed based on data from the previous observational trial of opioid usage. Using a 6-week mean prescription volume of 618 OMEs per patient with a standard deviation of 316 OMEs, at least 86 subjects in each group would be required to detect a difference with moderate effect size (approximately 25\% reduction in OMEs prescribed) in cumulative 6-week OME prescribing at an alpha of 0.05 and power of 0.9 . Six-month date ranges before and after the study were chosen to achieve a sample size of at least that magnitude based on historical trends. Descriptive statistics including proportions with percentages or means and standard deviations were performed as appropriate. Student $t$-tests and $\chi^{2}$ analysis were used to evaluate the impact of preawareness versus awareness group on the primary study outcomes. Multivariable main effects linear and logistic regression models incorporating baseline and treatment characteristics as well as preawareness versus awareness group were performed on the study outcomes using the 
Table 1. Baseline and Treatment Characteristics for Patients in Pre-awareness and Awareness Groups

\begin{tabular}{|c|c|c|c|}
\hline Factor & $\begin{array}{c}\text { Preawareness Proportion (\%) } \\
\text { or Mean (SD) }\end{array}$ & $\begin{array}{c}\text { Awareness Group Proportion (\%) } \\
\text { or Mean (SD) }\end{array}$ & $P$ Value \\
\hline Preawareness group & $129 / 129(100 \%)$ & $0 / 130(0 \%)$ & NA \\
\hline Age $(y)$ & $38.3(13.2)$ & $35.4(11.2)$ & .053 \\
\hline Caucasian & $110 / 129(85.3 \%)$ & $114 / 130(87.7 \%)$ & .57 \\
\hline Current smoking & $10 / 129(7.8 \%)$ & $9 / 130(6.9 \%)$ & .8 \\
\hline BMI $\left(\mathrm{kg} / \mathrm{m}^{2}\right)$ & $27(5.4)$ & $26.8(5.2)$ & .86 \\
\hline Operative time (min) & $160.2(42)$ & $160.2(47.1)$ & .99 \\
\hline American Society of Anesthesiologists I or II & $110 / 129(85.3 \%)$ & $118 / 130(90.8 \%)$ & .173 \\
\hline Resident prescribed initial opioid & $37 / 129(28.7 \%)$ & $67 / 130(51.5 \%)$ & $<.001$ \\
\hline Preoperative opioid usage & $59 / 129(45.7 \%)$ & $32 / 130(24.6 \%)$ & $<.001$ \\
\hline Postoperative aspirin & $114 / 129(88.4 \%)$ & $120 / 130(92.3 \%)$ & .28 \\
\hline Postoperative NSAID & $101 / 129(78.3 \%)$ & $123 / 130(94.6 \%)$ & $<.001$ \\
\hline
\end{tabular}

NOTE. Proportions or means (standard deviation or percentage). Boldface font indicates statistical significance.

NA, not available.

standard statistical package JMP Pro, version 14, by Statistical Analysis Software (Cary, NC). Statistical significance was taken at $P<.05$ in multivariable analyses.

\section{Results}

Baseline and treatment characteristics are shown in Table 1. Baseline characteristics were similar for the 2 groups. Treatment factors differed somewhat with the preawareness group having increased rates of preoperative opioid usage, decreased resident prescription of initial postoperative opioid, and decreased rate of postoperative NSAID prescription.

Table 2 displays unadjusted differences in cumulative 6-week OMEs prescribed, initial prescription size in OMEs, whether or not patients were prescribed a refill after their initial discharge opioid, and the total number of additional postoperative opioid prescriptions. Patients in the preawareness group were prescribed significantly more opioids by 6 weeks postoperatively, had a greater initial prescription size, and had more postoperative opioid prescriptions than patients in the awareness group.

Table 3 demonstrates the results of multivariable modeling of the impact of baseline and treatment factors on opioid-related outcomes. Preawareness group and preoperative opioid usage were associated with significantly greater additional cumulative 6-week OMEs prescribed while Caucasian race and postoperative NSAID were associated with decreased additional cumulative 6-week OMEs prescribed. Preawareness group and preoperative opioid usage were associated with increased additional initial discharge opioid prescription volume. Female sex and preoperative opioid usage were associated with increased odds of additional opioid prescription. Postoperative NSAID prescription was associated with decreased additional number of opioid prescriptions while preoperative opioid usage and bilateral procedures were associated with increased number of additional opioid prescriptions.

There were no returns to the emergency department or admissions for pain control in either group. Five of 130 patients $(3.8 \%)$ returned to the emergency department in the awareness group ( 2 pulmonary edema and 1 each hematuria, contact dermatitis, and medication reaction), whereas 1 of 129 patients $(0.8 \%)$ returned to the emergency department in the preawareness group (medication reaction) within 90 days postoperatively $(P=.100)$. One patient in the awareness group was admitted for pulmonary edema management within 90 days postoperatively. This resolved with medical management.

\section{Discussion}

In analyses that adjusted for baseline patient and operative characteristics, there was a significant reduction of cumulative 6-week OMEs (51.7\% reduction) prescribed

Table 2. Unadjusted Opioid Prescription Outcomes by Awareness vs Pre-awareness Group

\begin{tabular}{|c|c|c|c|}
\hline Initial OMEs prescribed & $559.9(385.5)$ & $359.7(356.3)$ & $<.001$ \\
\hline Any additional postoperative opioid prescriptions & $63 / 129(48.8 \%)$ & $41 / 130(31.5 \%)$ & .004 \\
\hline Number of additional postoperative opioid prescriptions & $0.9(1.2)$ & $0.5(1)$ & .007 \\
\hline
\end{tabular}

NOTE. Proportions or means (standard deviation or percentage). Boldface font indicates statistical significance. 


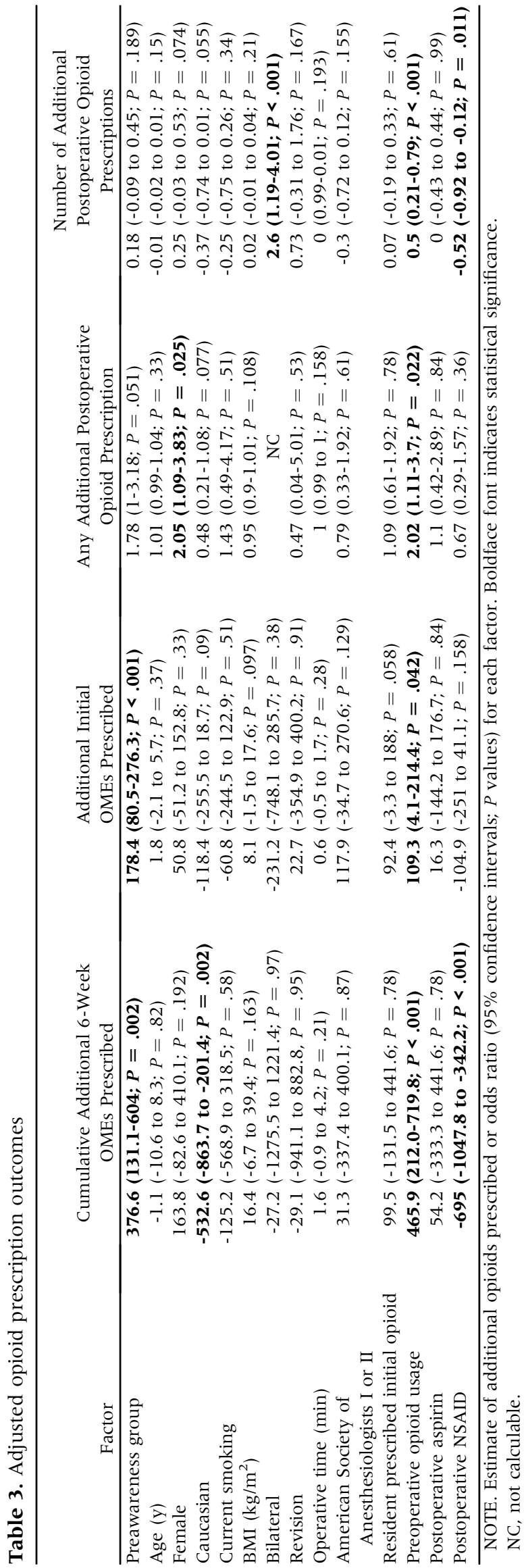

and initial OMEs prescribed ( $>35.8 \%$ reduction) in patients undergoing hip arthroscopy once providers had normative data on postoperative opioid consumption (awareness group). This confirmed the primary study hypothesis. The odds of additional opioid prescription did not significantly increase, which is in contrast to our secondary hypothesis. Although the mechanism of the reduction in opioid prescribing is likely multifactorial, prescriber awareness of normal opioid consumption could have reset prescribers' internal benchmarks for opioid prescribing and encouraged providers to set patient expectations regarding postoperative opioid prescriptions. These results before and after provider awareness of opioid usage trends suggest that knowledge of expected opioid consumption could have a significant impact on the volume of opioids prescribed.

There has been a recent emphasis on institutional and legislative efforts to decrease postoperative opioid prescribing. Stepan et al. ${ }^{19}$ demonstrated a 5.6 to 21.7 pill reduction in prescribing after elective orthopaedic procedures after a 1-hour educational session. Stanek et al. ${ }^{15}$ demonstrated a significant reduction in variability and volume of opioids prescribed after an educational intervention. Additionally, consensus-based guidelines have been recently published that advocate for low-volume opioid prescriptions ( 0 -20 oxycodone 5 -mg pills) after common surgical procedures including rotator cuff repair, anterior cruciate ligament reconstruction, and ankle fracture fixation. ${ }^{16}$ However, unlike the current study, none of these studies used direct observation of patient opioid usage to develop their guidelines. Individualized estimates of opioid overprescribing may be powerful metrics for prescribers.

Some states have also implemented mandatory prescription limits for postoperative pain. A recent report by Reid et al. $^{20}$ demonstrated that the volume of opioids prescribed by orthopaedic surgeons decreased by approximately one-half after the implementation of statewide legislation restricting early postoperative opioid prescriptions in Rhode Island. Our state enacted legislation limiting postoperative opioid prescribing on January 1,2018 . Because this could confound results, we performed a subgroup analysis that excluded these patients that underwent surgery on or after January 1, 2018 $(\mathrm{n}=61)$. Study results were similar and demonstrated a significant reduction in all opioid prescribing metrics in the awareness group compared to the preawareness group.

Preoperative opioid usage was significantly associated with increases in all postoperative opioid prescribing outcomes. In this study, the proportion of patients using opiates preoperatively was considerably decreased in the awareness group compared with the preawareness group (24.6\% compared with $45.7 \%$ ). Preoperative opioid usage has been associated with multiple adverse outcomes and increased postoperative opioid consumption in spine 
and fracture surgery, ${ }^{21-25}$ and has also been related to increased postoperative opioid consumption in hip arthroscopy. ${ }^{17}$ The prospective, observational study of opioid usage in patients undergoing hip arthroscopy demonstrated that patients with no preoperative opioid usage consumed approximately 20 oxycodone 5 -mg pills up to 6 weeks postoperatively compared with nearly 80 pills for patients with preoperative opioid usage. Prescribers in the current study indicated that they had started incorporating questions regarding for preoperative opioid usage during their history-taking and encouraged opioid cessation prior to surgery based at least in part on results of the previous study. This may account for the lower rate of preoperative opioid usage in the awareness group.

Postoperative NSAID prescription was associated with a significant decrease in cumulative opioid volume as well as decreased number of additional opioid prescriptions. The rate of postoperative NSAID prescribing was higher in patients in the awareness group $194.6 \%$ vs $78.3 \%$ ). Prescribers reported that they had increased the NSAID prescription rate resulting from data suggesting possible improvements in postoperative pain control with preoperative NSAID usage in a prospective, observational study of the early recovery period after hip arthroscopy. ${ }^{4}$ The opioid consumption reduction noted in this study is consistent with literature in anesthesiology associating NSAID usage with a $30 \%$ to $50 \%$ reduction in opioid consumption, a modest decrease in opioid-related side effects, and improvement in pain. ${ }^{26}$ As a possible added benefit, NSAIDs have been used commonly in hip arthroscopy to reduce the rate of postoperative heterotopic ossification ( $\mathrm{HO})$, which has a reported rate of up to $44 \% .{ }^{27} \mathrm{HO}$ prophylaxis using NSAIDs has been shown to reduce the rate of radiographic $\mathrm{HO} 4$-fold. However, $75 \%$ of $\mathrm{HO}$ is reported to be asymptomatic, and the side effects of NSAIDs can include gastrointestinal ulceration and renal injury. ${ }^{28}$ COX-2 selective inhibitors may reduce the rate of these side effects.

Caucasian race was significantly associated with decreased 6-week opioid prescribing along with a trend toward fewer additional postoperative opioid prescriptions. The reason for this association is unclear. Limited evidence has suggested an alternate trend towards decreased opiate prescription volume for nonCaucasian patients. ${ }^{29}$ The proportion of non-Caucasian patients is relatively low in this sample, and these results should be interpreted with caution. Females had higher odds of being prescribed an additional opioid during the 6-week postoperative period. Although the reason for this relationship is also unclear, prior reports have indicated increased opioid usage and higher rates of pain catastrophization in females. ${ }^{30,31}$

Resident involvement in the initial opioid prescription trended toward a significant association with greater initial opioid prescription volume. It is possible that prescribing residents were unaware of attending preferences regarding postoperative opioid prescriptions. Chiu et al. reported that surgical trainees rely almost exclusively on opioids for postoperative analgesia and do not often receive formal opioid-prescribing education. The authors encourage increased training on postoperative pain management in surgical training. ${ }^{32}$ Patients undergoing bilateral procedures had a higher number of postoperative opioid prescriptions, which is intuitive.

In conclusion, this study demonstrates that prescriber awareness of opioid consumption trends may help prescribers to reduce postoperative opioid prescription volume without significantly increasing opioid refill volume. We would encourage all surgeons to begin understanding their own patients' opioid usage and advocate for more research to help establish normative prescribing levels for many orthopaedic procedures. Improving rates of postoperative NSAID usage and reducing preoperative opioid usage may also have important roles in reduced postoperative opioid demand. Last, resident physicians may play an important role in appropriate opioid prescribing.

\section{Limitations}

This study has several limitations mainly related to its retrospective, observational nature. First, causation is difficult to determine in this study. Though prescribers significantly reduced their opioid prescriptions after the results of the prior observational study were known to them and readily report that this study had a major impact on their opioid prescribing, it is possible that the prescribers changed their practice because of other factors. However, we attempted to adjust for a variety of possible confounding factors, and determined sizeable differences in prescribing based on preawareness versus awareness group. Further providers still overprescribed opioids when judged by the prior study's mean opioid consumption (359.7 OMEs compared with 250 OMEs). This study also evaluates opioid prescriptions rather than opioid consumption. Although the purpose of the study was to evaluate prescriber behavior given prior norms, it would have been helpful to obtain additional details regarding patient usage. Patients in the awareness group had reduced rates of preoperative opioid usage and increased prescription of NSAIDs. This likely impacted postoperative opioid demand that could have otherwise driven increased refills and cumulative opioid prescriptions. However, additional subgroup analyses of these patients were performed in addition to the multivariable linear and logistic regression models with no change in overall study inference. Last, this study's before and after design lends itself to expectation bias. The influence of this bias is difficult to combat since the intervention did not permit blinding. 


\section{Conclusions}

Patients in the awareness group received significantly lower opioid volume without an increase in overall prescription numbers.

\section{References}

1. Griffin DR, Dickenson EJ, O'Donnell J, et al. The Warwick Agreement on femoroacetabular impingement syndrome (FAI syndrome): An international consensus statement. Br J Sports Med 2016;50:1169-1176.

2. Montgomery SR, Ngo SS, Hobson T, et al. Trends and demographics in hip arthroscopy in the United States. Arthroscopy 2013;29:661-665.

3. Colvin AC, Harrast J, Harner C. Trends in hip arthroscopy. J Bone Joint Surg Am 2012;94:e23.

4. Cunningham DJ, Lewis BD, Hutyra CA, Mather RC, Olson SA. Early recovery after hip arthroscopy for femoroacetabular impingement syndrome: a prospective, observational study. J Hip Preserv Surg 2017;4:299-307.

5. McCormick F, Nwachukwu BU, Alpaugh K, Martin SD. Predictors of hip arthroscopy outcomes for labral tears at minimum 2-year follow-up: The influence of age and arthritis. Arthroscopy 2012;28:1359-1364.

6. Morris BJ, Mir HR. The opioid epidemic: Impact on orthopaedic surgery. J Am Acad Orthop Surg 2015;23: 267-271.

7. Okie S. A flood of opioids, a rising tide of deaths. $N$ Engl $J$ Med 2010;363:1981-1985.

8. Rudd RA, Seth P, David F, Scholl L. Increases in drug and opioid-involved overdose deaths - United States, 20102015. MMWR Morb Mortal Wkly Rep 2016;65:1445-1452.

9. Kim N, Matzon JL, Abboudi J, et al. A prospective evaluation of opioid utilization after upper-extremity surgical procedures: Identifying consumption patterns and determining prescribing guidelines. J Bone Joint Surg Am 2016;98:e89.

10. O'Neil JT, Wang ML, Kim N, Maltenfort M, Ilyas AM. Prospective evaluation of opioid consumption after distal radius fracture repair surgery. Am J Orthop (Belle Mead NJ) 2017;46:E35-E40.

11. Hannon CP, Calkins TE, Li J, et al. The James A. Rand Young Investigator's Award: Large opioid prescriptions are unnecessary after total joint arthroplasty: A randomized controlled trial. J Arthroplasty 2019;34:S4-S10.

12. Helmerhorst GT, Vranceanu AM, Vrahas M, Smith M, Ring D. Risk factors for continued opioid use one to two months after surgery for musculoskeletal trauma. J Bone Joint Surg Am 2014;96:495-499.

13. Morris BJ, Zumsteg JW, Archer KR, Cash B, Mir HR. Narcotic use and postoperative doctor shopping in the orthopaedic trauma population. J Bone Joint Surg Am 2014;96:1257-1262.

14. Politzer CS, Kildow BJ, Goltz DE, Green CL, Bolognesi MP, Seyler TM. Trends in opioid utilization before and after total knee arthroplasty. J Arthroplasty 2018;33:S147-S153 e141.

15. Stanek JJ, Renslow MA, Kalliainen LK. The effect of an educational program on opioid prescription patterns in hand surgery: A quality improvement program. J Hand Surg Am 2015;40:341-346.
16. Overton HN, Hanna MN, Bruhn WE, et al. Opioid-prescribing guidelines for common surgical procedures: An expert panel consensus. J Am Coll Surg 2018;227: 411-418.

17. Cunningham D, Lewis B, Hutyra C, Nho S, Olson S, Mather R. Prospective, observational study of opioid use after hip arthroscopy for femoroacetabular impingement syndrome. Arthroscopy 2018;34:1488-1497.

18. von Elm E, Altman DG, Egger M, et al. The Strengthening the Reporting of Observational Studies in Epidemiology (STROBE) statement: Guidelines for reporting observational studies. Int J Surg 2014;12:1495-1499.

19. Stepan JG, Lovecchio FC, Premkumar A, et al. Development of an institutional opioid prescriber education program and opioid-prescribing guidelines: Impact on prescribing practices. J Bone Joint Surg Am 2019;101:5-13.

20. Reid DBC, Shah KN, Shapiro BH, Ruddell JH, Akelman E, Daniels AH. Mandatory prescription limits and opioid utilization following orthopaedic surgery. J Bone Joint Surg Am 2019;101:e43.

21. Pugely AJ, Bedard NA, Kalakoti P, et al. Opioid use following cervical spine surgery: Trends and factors associated with long-term use. Spine J 2018;18:1974-1981.

22. Lee D, Armaghani S, Archer KR, et al. Preoperative opioid use as a predictor of adverse postoperative self-reported outcomes in patients undergoing spine surgery. J Bone Joint Surg Am 2014;96:e89.

23. Jain N, Brock JL, Phillips FM, Weaver T, Khan SN. Chronic preoperative opioid use is a risk factor for increased complications, resource use, and costs after cervical fusion. Spine J 2018;18:1989-1998.

24. Armaghani SJ, Lee DS, Bible JE, et al. Preoperative opioid use and its association with perioperative opioid demand and postoperative opioid independence in patients undergoing spine surgery. Spine (Phila Pa 1976) 2014;39: E1524-E1530.

25. Bot AG, Bekkers S, Arnstein PM, Smith RM, Ring D. Opioid use after fracture surgery correlates with pain intensity and satisfaction with pain relief. Clin Orthop Relat Res 2014;472:2542-2549.

26. Elia N, Lysakowski C, Tramer MR. Does multimodal analgesia with acetaminophen, nonsteroidal antiinflammatory drugs, or selective cyclooxygenase-2 inhibitors and patient-controlled analgesia morphine offer advantages over morphine alone? Meta-analyses of randomized trials. Anesthesiology 2005;103:1296-1304.

27. Rath E, Sherman H, Sampson TG, Ben Tov T, Maman E, Amar E. The incidence of heterotopic ossification in hip arthroscopy. Arthroscopy 2013;29:427-433.

28. Yeung M, Jamshidi S, Horner N, Simunovic N, Karlsson J, Ayeni OR. Efficacy of nonsteroidal anti-inflammatory drug prophylaxis for heterotrophic ossification in hip arthroscopy: A systematic review. Arthroscopy 2016;32: 519-525.

29. Friedman J, Kim D, Schneberk T, et al. Assessment of racial/ethnic and income disparities in the prescription of opioids and other controlled medications in California. JAMA Intern Med 2019;179:469-476.

30. Serdarevic M, Striley CW, Cottler LB. Sex differences in prescription opioid use. Curr Opin Psychiatry 2017;30: 238-246. 
31. Keefe FJ, Lefebvre JC, Egert JR, Affleck G, Sullivan MJ, Caldwell DS. The relationship of gender to pain, pain behavior, and disability in osteoarthritis patients: The role of catastrophizing. Pain 2000;87:325-334.
32. Chiu AS, Healy JM, DeWane MP, Longo WE, Yoo PS. Trainees as agents of change in the opioid epidemic: Optimizing the opioid prescription practices of surgical residents. J Surg Educ 2018;75:65-71. 